\title{
Room temperature photo-response of titanium supersaturated silicon at energies over the bandgap
}

J. Olea ${ }^{1 *}$, E. López ${ }^{1}$, E. Antolín ${ }^{1}$, A. Martí ${ }^{1}$ and A. Luque ${ }^{1}$.

E. García-Hemme ${ }^{2,3,4}$, D. Pastor ${ }^{2,3,5}$, R. García-Hernansanz ${ }^{2,3}$, A. del Prado ${ }^{2,3}$ and G. González-Díaz ${ }^{2,3}$.

${ }^{1}$ Instituto de Energía Solar, E.T.S.I. Telecomunicación, Universidad Politécnica de Madrid, 28040 Madrid, Spain.

${ }^{2}$ Departamento de Física Aplicada III (Electricidad y Electrónica), Facultad de Ciencias Físicas, Universidad Complutense de Madrid, 28040 Madrid, Spain.

${ }^{3}$ CEI Campus Moncloa, UCM-UPM, 28040 Madrid, Spain.

${ }^{4}$ Materials Sciences Division, Lawrence Berkeley National Laboratory, 1 Cyclotron Road, Berkeley, California 94720, USA.

${ }^{5}$ School of Engineering and Applied Sciences, Harvard University. Cambridge Massachusetts 02138, USA.

*e-mail: j.olea@upm.es

\begin{abstract}
Silicon samples were implanted with high Ti doses and subsequently processed with the pulsed-laser melting (PLM) technique. The electronic transport properties in the $15-$ $300 \mathrm{~K}$ range and the room temperature spectral photoresponse at energies over the bandgap were measured. Samples with Ti concentration below the insulator-metal (I-M) transition limit showed a progressive reduction of the carrier lifetime in the implanted layer as Ti dose is increased. However, when the Ti concentration exceeded this limit, an extraordinary recovery of the photoresponse was measured. This result supports the theory of intermediate band (IB) materials and is of extreme importance for photovoltaic cells and Si-based detectors.
\end{abstract}




\section{INTRODUCTION}

The field of supersaturated Si has recently attracted much attention due to the unusual and interesting properties of these materials, with practical applications in photovoltaic devices [1, 2] and infrared (IR) detection [3-5]. High absorption coefficient and photoresponse in the IR $[6,7]$ could place silicon in the market as an option for imaging or security. For decades, IR detectors based on Si have been searched for, in order to integrate the device and the readout circuit. An IR detector working at room temperature (RT) and compatible with CMOS fabrication routes would drastically decrease the price of devices and applications [8].

Of especial interest is the link between $\mathrm{Si}$ supersaturated materials and the intermediate band (IB) theory [9]. It is very well known that transition metals and specially Ti are a source of deep levels when they are incorporated to crystalline silicon substrates. These levels actuate as fast recombination centers degrading the device performance. However, the classical understanding of recombination produced by deep levels is now called into question [10]. It has been reported that once the impurity concentration surpasses the insulator-metal (I-M) transition, deep levels associated to the impurities could form a band inside the bandgap. The presence of this band could change radically the application of the multi-phonon recombination theory. This supersaturated material could have a reduced non-radiative recombination, while in the case of a low impurity concentration these impurities would act just as lifetime killers. Although some authors reject the lifetime recovery predicted by the intermediate band theory [11], this recovery has been experimentally measured for $\mathrm{Ti}$ supersaturated Si at concentrations over the I-M transition limit [12], being this limit estimated to be over $10^{20} \mathrm{~cm}^{-3}[13]$.

Although the measurement of sub-bandgap photoresponse of supersaturated $\mathrm{Si}$ materials would give information on the feasibility of high efficient photovoltaic devices and IR photodetectors, and it has been recently the topic of several papers [14, 15], it is also necessary to analyze the optoelectronic properties of such materials at energies over the band gap. First, a device with enhanced properties below the bandgap could have deterioration over the band gap (as in Ref. [2]), and second, measurements in the visible and UV range could yield new and valuable information for the assessment of the material and the device.

In this work we analyze the I-M transition by the characterization of $\mathrm{Ti}$ supersaturated $\mathrm{Si}(\mathrm{Si}: \mathrm{Ti}$ ) layers fabricated by $\mathrm{Ti}$ ion implantation on $\mathrm{Si}$ and pulsed-laser melting (PLM). We measured the RT spectral sheet photoconductance (SSPC) of samples with Ti concentration below and over the I-M transition limit at wavelengths in the $200-1400 \mathrm{~nm}$ range and we connect the results with the electronic transport properties of the material. Results would point to a reduction in the non-radiative recombination once the I-M transition limit is well surpassed. 


\section{EXPERIMENTAL}

Silicon samples $1 \times 1 \mathrm{~cm}^{2}$ in size, grown in the (100) direction, single side polished, with a thickness of $525 \mu \mathrm{m}$, with resistivity $\rho \approx 3500 \Omega \mathrm{cm}\left(\mathrm{n} \approx 1.3 \times 10^{12} \mathrm{~cm}^{-3}\right)$, n-type, were implanted through the polished surface at RT with ${ }^{48} \mathrm{Ti}^{+}$at $32 \mathrm{keV}$ with $10^{13}, 10^{14}, 10^{15}$ and $10^{16} \mathrm{~cm}^{-2}$ doses with a Varian CF3000 refurbished ion implanter. Implantations were performed at $7^{\circ}$ tilt angle to reduce channeling effects. Subsequently, samples were PLM processed at RT in air with a $\operatorname{KrF}(248 \mathrm{~nm})$ excimer laser at an energy density of $1.4 \mathrm{~J} / \mathrm{cm}^{2}$. PLM processes were carried out at I.P.G. Photonics (New Hampshire, USA), and to process the samples, the homogenized laser spot $\left(1 \times 1 \mathrm{~mm}^{2}\right)$ was scanned over the whole surface overlapping the steps $10 \mu \mathrm{m}$ or less. The duration of the pulse was $20 \mathrm{~ns}$ and only one pulse was fired at each location. With this laser process we guarantee a good crystal quality of the resulting layers $[6,14,16]$.

Time-of-flight secondary ion mass spectrometry (ToF-SIMS) was carried out after the PLM process for all the samples to obtain the Ti depth profile. Measurements were obtained with an ION-TOF ToF-SIMS IV model with a $25 \mathrm{keV}$ pulsed $\mathrm{Bi}^{3+}$ beam at $45^{\circ}$ of incidence. A $10 \mathrm{kV}$ voltage was used to extract the secondary ions, and their time of flight from the sample to the detector was measured with a reflection mass spectrometer. Concentration profiles were calibrated by matching the dose to the $\mathrm{Ti}$ content in an implanted but not PLM processed sample, and the depth was calculated by optical profilometry measurement of the sputtered crater and assuming a constant erosion rate.

Samples reflectance was measured with a Perkin-Elmer Lambda-9 spectrophotometer in the $250-1000 \mathrm{~nm}$ wavelength range in order to detect possible changes in the implanted and PLM processed surfaces.

We used the van der Pauw geometry for the measurements of the electronic and optoelectronic properties of the samples. To this purpose $200 \mathrm{~nm}$ thick triangular Al contacts were evaporated at the four corners of the samples using the e-beam technique. A current of $1 \mathrm{~mA}$ was used to feed the contacts for all the measurements of the electronic transport and optoelectronic properties and a 0.8 T magnetic flux density was used for Hall effect characterization. A Keithley 4200 SCS model with four source and measure units was used to obtain the electronic transport properties. To minimize the uncertainty in the measurements all configurations were averaged, changing also the current and magnetic field direction. Sheet resistance and Hall effect was measured from $14 \mathrm{~K}$ to RT. Samples were placed in a closed-cycle Janis cryostat attached to a turbomolecular pump.

To characterize the SSPC of fabricated samples, spectral photoconductance $\Delta G_{S}$ was measured at RT in the $200-1400 \mathrm{~nm}$ optical range with the van der Pauw set-up, as in Ref. 14, obtaining significant results at wavelengths below $1240 \mathrm{~nm}$. Samples were illuminated by the implanted surface. A $1 / 4 \mathrm{~m}$ optical path monochromator fed with 
a $95 \mathrm{~W}$ halogen lamp was used as illumination source for the complete measurement. Optical filters on a wheel blocked the monochromator's secondaries throughout the whole range. With this arrangement a power density of around $2 \times 10^{-6} \mathrm{~W} / \mathrm{cm}^{2}$ was obtained in almost the full range. Two calibrated detectors were used to normalize the measurements to the spectral power density $I_{0}$ : a Si detector for the $200-940 \mathrm{~nm}$ range and a $\mathrm{Ge}$ detector for the $950-1400 \mathrm{~nm}$ range. Measurements were carried out chopping the light beam at $23.5 \mathrm{~Hz}$ and the voltage developed was measured with a SR830 digital signal processing lock-in amplifier manufactured by Stanford Research Systems. RT electronic transport properties were used to reference the SSPC, $\Delta G_{S} / I_{0}$, which will be presented in $\Omega^{-1} \mathrm{~s} \mathrm{~cm}^{2}$ units. 


\section{RESULTS}

Figure 1 shows the results of the sheet resistance measurements for the $\mathrm{Ti}$ implanted and PLM processed samples as a function of the temperature. Samples implanted with $10^{13}$ and $10^{14} \mathrm{~cm}^{-2}$ Ti doses present a sheet resistance undistinguishable from an unimplanted reference sample (not shown for clarity). From about 50 - $60 \mathrm{~K}$ to $300 \mathrm{~K}$, samples show a potential increase of the sheet resistance that is typically related to phonon scattering processes in silicon. At temperatures below $50 \mathrm{~K}$ a strong increase in the sheet resistance can be seen. This increase is produced by the freeze-out process. The inset of figure 1 shows the $\mathrm{Ti}$ depth profile of the same samples. The sample implanted with a $10^{13} \mathrm{~cm}^{-2}$ dose has a Ti concentration well below the I-M transition limit, and the Ti peak in the sample implanted with a $10^{14} \mathrm{~cm}^{-2}$ dose is very close to this limit. For higher implantation doses the electrical behavior is different and it will be discussed in the next section. Note that Ti depth profile of samples implanted with $10^{15}$ and $10^{16} \mathrm{~cm}^{-2}$ Ti doses clearly surpasses the I-M transition limit of $\mathrm{Ti}$ in $\mathrm{Si}$ in layer thicknesses of about 15 and $75 \mathrm{~nm}$, respectively.

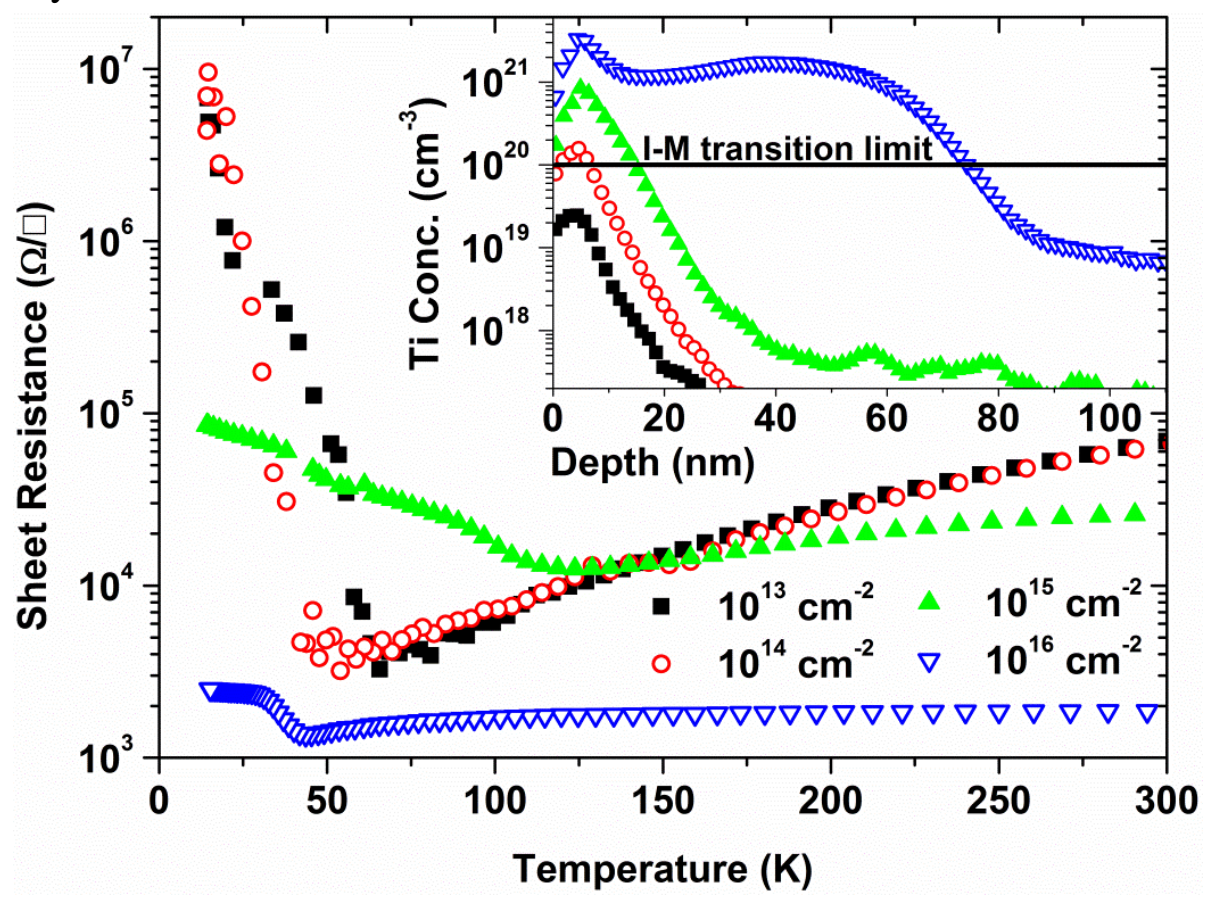

Figure 1

In Fig. 2 we present the main results of the SSPC measurements for all the implanted doses and also for a reference unimplanted sample. Simulation curves (continuous lines) for all the samples (except for the sample implanted with a $10^{16} \mathrm{~cm}^{-2}$ $\mathrm{Ti}$ dose) are also shown and will be discussed in the next section. The onset of the $\mathrm{Si}$ bandgap is shown for all the samples at about $1200 \mathrm{~nm}$. At higher wavelengths no measurable signal is obtained. In comparing the different samples, it can be seen that the reference sample has the highest SSPC in the whole wavelength range. This SSPC is 
almost flat, with a small dip at about $375 \mathrm{~nm}$ that corresponds to the opposite feature in the reflectance of Si [17]. The sample implanted with the $10^{13} \mathrm{~cm}^{-2} \mathrm{Ti}$ dose has a reduced SSPC but with the same shape. SSPC is progressively reduced for samples implanted with $10^{14}$ and $10^{15} \mathrm{~cm}^{-2}$ Ti doses and the shape is changing, decreasing its magnitude for low wavelengths. In contrast, the sample implanted with the $10^{16} \mathrm{~cm}^{-2} \mathrm{Ti}$ dose has a recovered SSPC, almost equal to the reference, except for low wavelengths. Interestingly, below $400 \mathrm{~nm}$ an abrupt fall of the sheet conductance is shown, reaching the noise level at about $380 \mathrm{~nm}$.

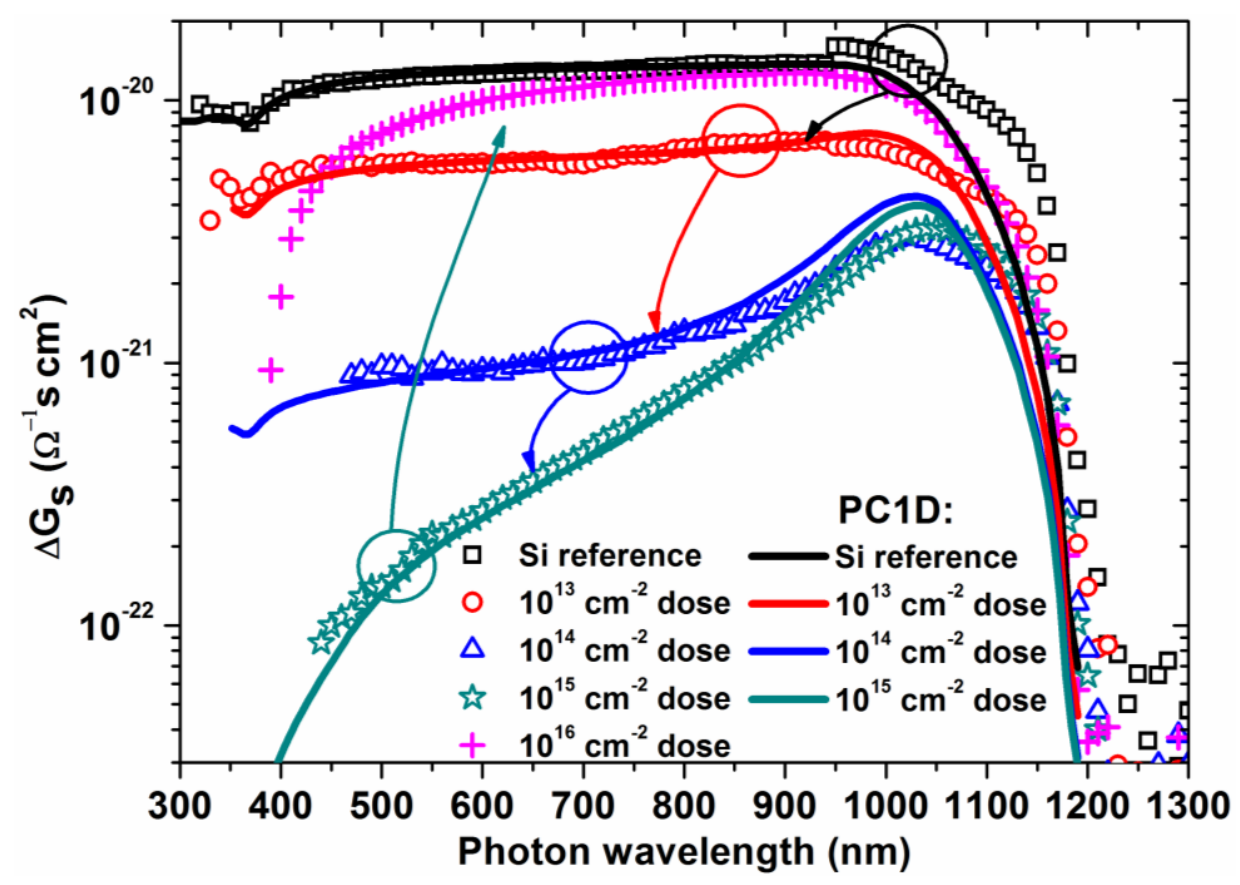

Figure 2 


\section{DISCUSSION}

Samples implanted with $10^{13}$ and $10^{14} \mathrm{~cm}^{-2}$ doses do not present significative differences in the sheet resistance in comparison with an unimplanted reference sample. This could mean that although the Ti concentration is high, in fact around five orders of magnitude over the solid solubility limit of $\mathrm{Ti}$ in $\mathrm{Si}$ [18], the influence of the implanted layer on the effective sheet resistance is negligible. If we consider that $\mathrm{Ti}$ could introduce deep donor levels typically at about $0.3 \mathrm{eV}$ below the conduction band [19], and that in $\mathrm{Ti}$ supersaturated $\mathrm{Si}$ only around $1 \%$ or below of the Ti atoms are substitutional [20], even with the high concentration introduced, the electron concentration would be relatively low. From Fig. 1 we obtain $2 \times 10^{20} \mathrm{~cm}^{-3}$ Ti atoms for the sample implanted with the $10^{14} \mathrm{~cm}^{-2}$ dose. This would mean around $10^{15} \mathrm{~cm}^{-}$ ${ }^{3}$ electrons ionized from the $0.3 \mathrm{eV}$ level at room temperature. This is 1000 times higher than the electron concentration in the substrate (around $1.3 \times 10^{12} \mathrm{~cm}^{-3}$ ), while the substrate/implanted layer thicknesses ratio would be around 50000 times. Even taking into account similar values for the mobility, conduction by the substrate would dominate. We suggest that I-M transition limit has not been surpassed in these samples, what is consistent with the location of this limit over $10^{20} \mathrm{~cm}^{-3}$ for Ti in Si (see inset in Fig. 1) [13].

However, the sample implanted with the $10^{15} \mathrm{~cm}^{-2}$ dose shows a sheet resistance higher than the sheet resistance of the samples implanted with lower doses in the $60-$ $125 \mathrm{~K}$ range. Since as quoted above, the sheet resistance of low dose samples represent also the sheet resistance of the substrate, this means that in this range conduction is mainly taking place in the implanted layer. Because two layers in parallel (implanted layer and substrate) would have a lower sheet resistance than any of the layers alone and we are only contacting the implanted layer, we conclude that there is an electrical isolation between the implanted layer and the substrate. This isolation is characteristic of $\mathrm{Ti}$ supersaturated $\mathrm{Si}$ and has been analyzed and modeled elsewhere [21]. The minimum in the sheet resistance characteristic indicates the temperature of the onset of isolation. At temperatures over $125 \mathrm{~K}$ both implanted layer and substrate are conducting in parallel and, as temperature decreases from this minimum, conduction takes place more and more by the implanted layer. Contrary to the behavior of samples implanted with lower doses, the freeze out effect is not present in the $10^{15} \mathrm{~cm}^{-2}$ dose sample and the sheet resistance at low temperature is relatively low.

Finally, the sample implanted with the highest dose presents also the typical temperature behavior of a $\mathrm{Ti}$ supersaturated Si bilayer, with a minimum indicating the isolation effect. Besides, the sheet resistance of this sample does not cross the sheet resistance of the substrate, not even in the isolation range at temperatures below $50 \mathrm{~K}$, indicating that the carrier concentration in the implanted layer is very high. Hall effect measurements at low temperatures point to a carrier concentration in the $10^{21} \mathrm{~cm}^{-3}$ range for the Si:Ti layer, similarly to the Ti concentration in the $75 \mathrm{~nm}$ thick layer obtained by ToF-SIMS, being consistent with the formation of a semi-filled IB. Moreover, the freeze out effect at low temperatures is not present in this sample, what is consistent 
with the high carrier concentration found [22]. As a consequence, an I-M transition in samples implanted with $10^{15}$ and $10^{16} \mathrm{~cm}^{-2}$ Ti doses is suggested.

Regarding the variation of the SSPC as a function of the Ti implanted dose presented in Fig. 2, it can be seen how the magnitude decreases when the dose is increased from $10^{13} \mathrm{~cm}^{-2}$ to $10^{15} \mathrm{~cm}^{-2}$. This behavior is consistent with the traditional theory of recombination introduced by the so-called lifetime killers [23]: the more $\mathrm{Ti}$ impurities, the more recombination. Also, for the reference sample and the sample implanted with the lowest Ti dose, the SSPC curve is almost flat in the whole spectral range, while for higher doses the value of the SSPC decreases at lower wavelengths. This result is consistent again with the introduction of strong lifetime killers at the illuminated surface. The effect of the $\mathrm{Ti}$ impurities would be stronger at lower wavelengths at which $\mathrm{Si}$ has a higher absorption coefficient, and much more photons are absorbed near the surface, increasing the recombination. However, for the highest dose implanted the SSPC increases, giving a result that seems to be in agreement with the IB theory [10]. The sample implanted with the highest Ti dose has a SSPC curve that mixes both behaviors commented: it is almost flat over a certain wavelength range and abruptly decreases at lower wavelengths. Since this fall of the SSPC curve at about $400 \mathrm{~nm}$ could be produced by an increase of the reflectance of the new material created after Ti implantation and PLM processing, reflectance of this sample was measured and it is presented in Fig. 3. This figure shows the ratio of the reflectance of the sample implanted with the $10^{16} \mathrm{~cm}^{-2}$ Ti dose versus the reflectance of the silicon reference substrate. As it can be seen, the increase of the reflectance of the implanted sample is lower than $10 \%$ in the whole range. This small variation of the reflectance cannot be the origin of the abrupt fall at about $400 \mathrm{~nm}$ in the SSPC of this sample. Moreover, in the wavelength region where the fall occurs, the ratio of the reflectances tends to unity. Consistently with the measurement presented in Fig. 3, the sample implanted with a $10^{16} \mathrm{~cm}^{-2}$ Ti dose appears with a dark green color to the naked eye, what would correspond to the peak at about $500 \mathrm{~nm}$. Reflectance of samples implanted with lower doses show even a lower increase. 


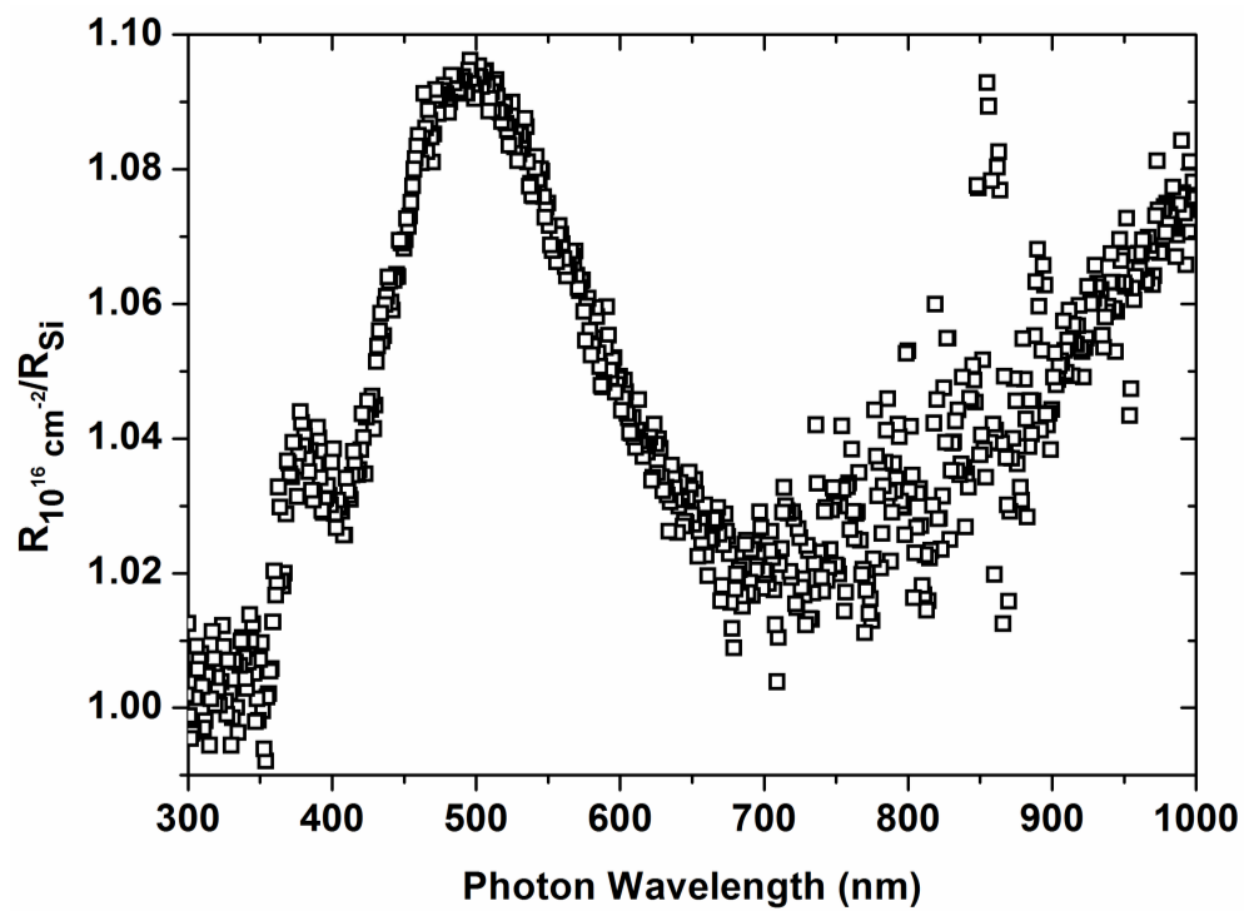

Figure 3

To get insight into these results we modeled the SSPC of the samples with a simple model in the PC1D framework [24]. For the reference substrate, a $525 \mu \mathrm{m}$ layer with the properties of the substrate described in the experimental section was configured, having as parameters the bulk lifetime $\tau_{\text {вик }}$, and the front (illuminated) and rear surface recombination velocities, $S_{\text {FRONT }}$ and $S_{\text {REAR }}$, respectively. For the implanted samples a thin superficial layer was also simulated to account for the variation of the properties in the $\mathrm{Ti}$ implanted layer. The parameters used for the implanted layer were the thickness, the lifetime, the electron concentration and the electron mobility, being in all cases the holes mobility 3 times lower than the electron mobility. Effects of the recombination velocity of the surface were included in the reduced value of the lifetime in the thin implanted surface layer. Although the implanted layer is supposed to have an IB at high Ti concentrations, we are unable to simulate a three bands semiconductor properly, and therefore we chose to not include the sample implanted with the highest dose in the spectral simulations. Regarding the mobility values simulated, we realized that mobility can be varied in a very broad range without affecting the results. The reason is that since the layers are very thin, most of the light is absorbed in the bulk, and therefore the SSPC in these layers is negligible. Due to the low thickness, implanted layers are acting just as a recombination surfaces.

In PC1D the power spectral density was configured to be constant with a $2 \times 10^{-6}$ $\mathrm{W} / \mathrm{cm}^{2}$ in the $250-1200 \mathrm{~nm}$ wavelength range, similarly to the experimental configuration (see Figure 4.a). 

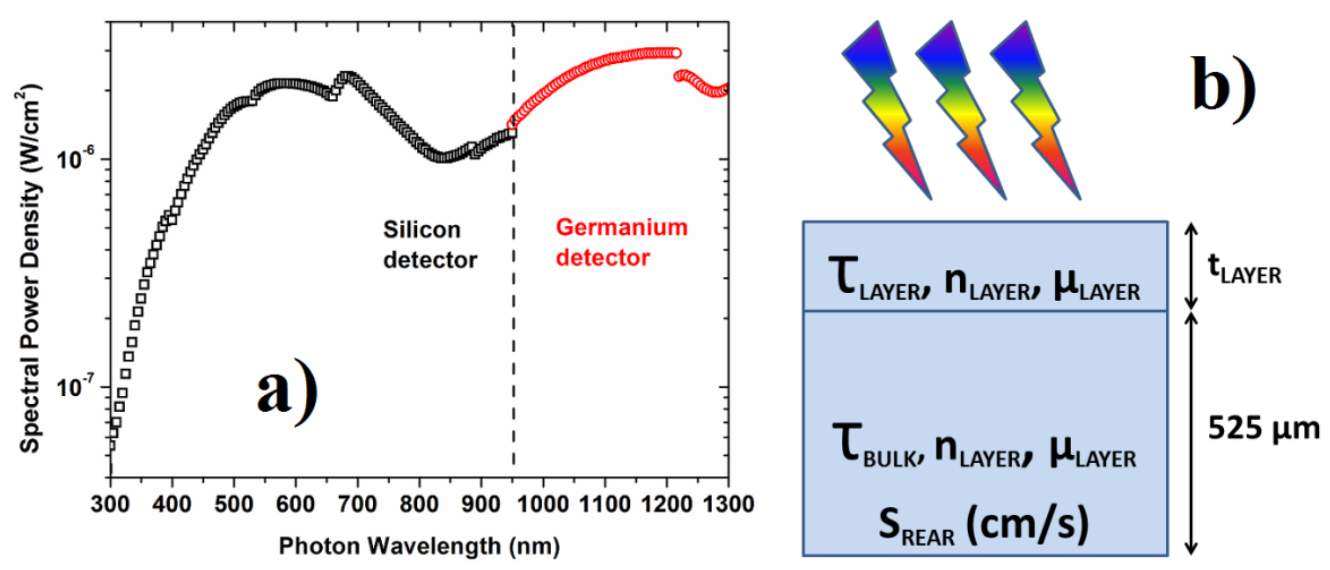

Figure 4

Other parameters such as absorption coefficient or reflectance of silicon were configured with their standard values. The magnitude simulated was the cumulative excess conductivity integrated in the whole thickness of the device for every wavelength to yield the SSPC:

$$
\Delta G_{S}(\lambda)=\int_{0}^{525} \Delta \sigma_{i l}(\lambda, t) d t
$$

where $\Delta G_{S}$ is the increase of the sheet conductance and $\Delta \sigma_{i l}$ is the increase in conductivity under illumination.

A diagram that summarizes the simulations is shown in Fig. 4.b, and Table 1 has the values of the parameters used for the simulations presented in the same figure. For the fitting process values for $\tau_{\text {BULK }}$ and $S_{\text {REAR }}$ were first established. Then, $S_{\text {FRONT }}$ for the reference sample and $\tau_{\text {LAYER }}$ for the samples implanted with $10^{13}$ and $10^{14} \mathrm{~cm}^{-2}$ Ti doses were adjusted. For the samples implanted with $10^{13}$ and $10^{14} \mathrm{~cm}^{-2} \mathrm{Ti}$ doses, the thickness used was calculated from the ToF-SIMS profile, obtaining the thickness that contains the $90 \%$ of the Ti remaining dose. For the samples implanted with $10^{15}$ and $10^{16} \mathrm{~cm}^{-2} \mathrm{Ti}$ doses the thickness used was the thickness where the Ti concentration surpassed the I-M transition limit.

For the simulations presented in Fig. 2, the values for $\tau_{\text {BULK }}, S_{\text {REAR }}$ and $S_{\text {FRONT }}$ were $500 \mu \mathrm{s}, 6000 \mathrm{~cm} / \mathrm{s}$ and $150 \mathrm{~cm} / \mathrm{s}$, respectively. These values could be varied in a relatively short range yielding similar results with the same conclusions. For example, the bulk lifetime could be varied in the $\tau_{\text {виL }}>100 \mu$ s range, but below this value the fitting was not possible. Since the rear surface was not modified by the implantation, $\mathrm{S}_{\text {REAR }}$ was maintained constant for all the samples. For a substrate with $\mathrm{n} \approx 1.3 \times 10^{12} \mathrm{~cm}^{-3}$, $\tau_{\text {вицк }}$ values in the $0.1-1 \mathrm{~ms}$ range are realistic [25].

For the fitting of the Si reference sample quite low values of $S_{\mathrm{FRONT}}$ were used. Also, considering that the rear surface is not polished, a value of $6000 \mathrm{~cm} / \mathrm{s}$ for $S_{\text {REAR }}$ might be unrealistically low [26]. However, with the extremely low carrier 
concentration of this substrate, surface states could be bending the band quite strongly, preventing carriers from getting close to the surface and reducing the effective surface recombination velocities even without passivation [27].

Table 1 shows the results for the lifetime of the implanted layer that yielded the simulated SSPC presented in Fig. 2. As it can be seen the simulated SSPC fits to the measured curves in the whole spectral range, pointing to the consistency of the model presented. Lifetime values for the implanted layer follow the expected trend in the scope of the classical theory: lifetime is reduced when the $\mathrm{Ti}$ dose is increased whenever the Ti concentration is below the I-M transition limit $[23,9]$. Also, for lower values of $\tau_{\text {LAYER }}$ recombination is stronger at low wavelengths since a high absorption coefficient would make generation to occur mostly at the surface. This hypothesis is consistent with the ToF-SIMS figures of samples implanted with Ti doses of $10^{13}$ and $10^{14} \mathrm{~cm}^{-2}$ since Ti concentration in these samples does not surpass the I-M transition limit (see inset in Fig. 1). Moreover, the electronic transport properties of these samples do not differ from the reference substrate, indicating that $\mathrm{Ti}$ impurities are just acting as deep levels producing a very low carrier concentration at RT. Taking into account these results we can consider the sample implanted with the $10^{14} \mathrm{~cm}^{-2}$ dose to be just below the I-M transition limit.

\begin{tabular}{|c|c|c|c|}
\hline & $\mathrm{t}_{\text {LAYYR }}(\mathrm{nm})$ & $\mathrm{n}_{\text {LAYER }}\left(\mathrm{cm}^{-3}\right)$ & $\tau_{\text {LAYER }}(\mathrm{ns})$ \\
\hline $10^{13} \mathrm{~cm}^{-2}$ & 12.5 & $1.3 \times 10^{12}$ & 2 \\
\hline $10^{14} \mathrm{~cm}^{-2}$ & 10.1 & $1.3 \times 10^{12}$ & 0.18 \\
\hline $10^{15} \mathrm{~cm}^{-2}$ & 15 & $3-5 \times 10^{12}$ & $2-10 \times 10^{-3}$ \\
\hline
\end{tabular}

Table 1

The sample implanted with a $10^{15} \mathrm{~cm}^{-2}$ dose has a Ti concentration clearly over the I-M transition limit in a very thin layer and the electronic transport properties are also consistent with the overcoming of this limit [21]. In the $1000-1170 \mathrm{~nm}$ range this sample has SSPC values higher than the sample implanted with the $10^{14} \mathrm{~cm}^{-2}$ Ti dose. This cannot be explained if we assume the classical theory of recombination, since with more Ti concentration recombination should be higher at all wavelengths. We suggest that although the I-M transition limit has been surpassed and a certain recovery of the lifetime is expected, the recombination in the tails of the implantation produces a detrimental effect that reduces the effective lifetime of the implanted layer. Moreover, since the implanted layer thickness is very low, and the Ti concentration is closer to the I-M transition limit, inhomogeneities could produce areas with a Ti concentration below this limit.

Although we were unable to simulate the results of the SSPC for the sample implanted with the $10^{16} \mathrm{~cm}^{-2} \mathrm{Ti}$ dose, it is very interesting to note that once the I-M transition limit is well surpassed, there is a recovery in the SSPC values, almost reaching the curve of the reference substrate. This result cannot be explained by the classical theory and is consistent with the IB theory. Regarding the abrupt fall at 400 $\mathrm{nm}$, we propose that once the absorption coefficient is high enough, almost all photons are absorbed in the implanted region, increasing the recombination and reducing the 
SSPC below the measurement noise level. For silicon, when the photon wavelength is around $400 \mathrm{~nm}$, the absorption coefficient is approximately $10^{5} \mathrm{~cm}^{-1}$ being the absorption length less than $100 \mathrm{~nm}$. This explanation is consistent with ToF-SIMS measurements and the SSPC of the sample implanted with the $10^{16} \mathrm{~cm}^{-2} \mathrm{Ti}$ dose.

Finally, previously reported measurements of $\mathrm{Si}: \mathrm{Ti}$ materials and devices showed an increase of the photoresponse at energies below the bandgap [4, 28] with a small reduction over the bandgap [2], consistently with the measurements presented here. These results could be interesting for photovoltaic applications and for visible extended to IR photodetectors. 


\section{CONCLUSIONS}

We have reported RT measurements of SSPC at energies over the bandgap of 4 samples implanted with $\mathrm{Ti}$ concentration below and above the I-M transition limit. Electronic transport properties of samples are consistent with the ToF-SIMS results, showing a temperature behavior that has been previously modeled and the absence of freeze out for samples implanted with a concentration over the I-M transition limit. Classical behavior of $\mathrm{Ti}$ as a lifetime killer is obtained when the Ti concentration is below the I-M transition limit, reducing progressively the SSPC. Once the I-M transition is well surpassed, SSPC is recovered, having values comparable to the ones of the reference sample without Ti implantation. These suggest the reduction of the nonradiative recombination once the I-M transition limit is surpassed.

\section{ACKNOWLEDGEMENTS}

Authors would like to acknowledge the C. A. I. de Técnicas Físicas of the Universidad Complutense de Madrid for the ion implantations and metallic evaporations, the Nanotechnology and Surface Analysis Services of the Universidad de Vigo C.A.C.T.I. for ToF-SIMS measurements and Dr. Jose Pablo González García from CIEMAT for reflectance measurements. Research by E. García-Hemme was supported by a PICATA predoctoral fellowship of the Moncloa Campus of International Excellence (UCM-UPM). J. Olea acknowledges financial support from the MICINN within the program Juan de la Cierva (JCI-2011-10402), under which this research was undertaken. D. Pastor acknowledges the financial support to the grant EX-2010-0662 from the Spanish Science Ministry. This work was partially supported by the Spanish MINECO (Economic and Competitiviness Ministery) under grant TEC 2013-41730-R. The authors acknowledge the financial support from la Comunidad de Madrid through the funding of the project MADRID-PV (S2013/MAE-2780). 


\section{FIGURE CAPTIONS}

Figure 1. Sheet resistance as a function of measured temperature of four Si samples implanted with different Ti doses $\left(10^{13}, 10^{14}, 10^{15}\right.$ and $\left.10^{16} \mathrm{~cm}^{-2}\right)$ after a PLM process. The inset shows the Ti concentration depth profile of the same samples obtained by ToF-SIMS.

Figure 2. SSPC in the $300-1300 \mathrm{~nm}$ optical range of four silicon samples implanted with an increasing Ti dose, from $10^{13} \mathrm{~cm}^{-2}$ to $10^{16} \mathrm{~cm}^{-2}$, and PLM processed, measured with the van der Pauw set up at room temperature. SSPC of a reference unimplanted sample is also shown. Solid lines show the simulated curves using PC1D as described in the text.

Figure 3. Ratio of the reflectance of the sample implanted with the $10^{16} \mathrm{~cm}^{-2} \mathrm{Ti}$ dose and the reflectance of the silicon reference sample, as a function of the incident photon wavelength.

Figure 4. a) Spectral power density of the complete optical set-up used for the characterization of the spectral sheet photoconductance, including the $95 \mathrm{~W}$ halogen lamp and the monochromator. b) Diagram of the structure simulated with PC1D.

Table 1. Values of parameters used for the simulations presented in Fig. 2. 


\section{REFERENCES}

[1] A. Luque, A. Marti, and C. Stanley, "Understanding intermediate-band solar cells," Nature Photonics, vol. 6, no. 3, pp. 146-152, Mar, 2012.

[2] S. Silvestre, A. Boronat, M. Colina, L. Castaner, J. Olea, D. Pastor, A. del Prado, I. Martil, G. Gonzalez-Diaz, A. Luque, E. Antolin, E. Hernandez, I. Ramiro, I. Artacho, E. Lopez, and A. Marti, "Sub-Bandgap External Quantum Efficiency in Ti Implanted Si Heterojunction with Intrinsic Thin Layer Cells," Japanese Journal of Applied Physics, vol. 52, no. 12, Dec, 2013.

[3] J. P. Mailoa, A. J. Akey, C. B. Simmons, D. Hutchinson, J. Mathews, J. T. Sullivan, D. Recht, M. T. Winkler, J. S. Williams, J. M. Warrender, P. D. Persans, M. J. Aziz, and T. Buonassisi, "Room-temperature sub-band gap optoelectronic response of hyperdoped silicon," Nature Communications, vol. 5, Jan, 2014.

[4] E. Garcia-Hemme, R. Garcia-Hernansanz, J. Olea, D. Pastor, A. del Prado, I. Martil, and G. Gonzalez-Diaz, "Sub-bandgap spectral photo-response analysis of Ti supersaturated Si," Applied Physics Letters, vol. 101, no. 19, Nov 5, 2012.

[5] X. Dong, N. Li, Z. Zhu, H. Shao, X. Rong, C. Liang, H. Sun, G. Feng, L. Zhao, and J. Zhuang, "A nitrogen-hyperdoped silicon material formed by femtosecond laser irradiation," Applied Physics Letters, vol. 104, no. 9, Mar 3, 2014.

[6] J. Olea, A. del Prado, D. Pastor, I. Martil, and G. Gonzalez-Diaz, "Sub-bandgap absorption in Ti implanted Si over the Mott limit," Journal of Applied Physics, vol. 109, no. 11, Jun 1, 2011.

[7] C. B. Simmons, A. J. Akey, J. P. Mailoa, D. Recht, M. J. Aziz, and T. Buonassisi, "Enhancing the Infrared Photoresponse of Silicon by Controlling the Fermi Level Location within an Impurity Band," Advanced Functional Materials, vol. 24, no. 19, pp. 2852-2858, May, 2014.

[8] Photonics PPP Multi Annual Roadmap "Towards 2020 - Photonics Driving Economic Growth in Europe", 77 - 81, (2013).

[9] A. Luque, and A. Marti, "Increasing the efficiency of ideal solar cells by photon induced transitions at intermediate levels," Physical Review Letters, vol. 78, no. 26, pp. 5014-5017, Jun 30, 1997.

[10] A. Luque, A. Marti, E. Antolin, and C. Tablero, "Intermediate bands versus levels in non-radiative recombination," Physica B-Condensed Matter, vol. 382, no. 1-2, pp. 320-327, Jun 15, 2006.

[11] J. J. Krich, B. I. Halperin, and A. Aspuru-Guzik, "Nonradiative lifetimes in intermediate band photovoltaics-Absence of lifetime recovery," Journal of Applied Physics, vol. 112, no. 1, Jul 1, 2012.

[12] E. Antolin, A. Marti, J. Olea, D. Pastor, G. Gonzalez-Diaz, I. Martil, and A. Luque, "Lifetime recovery in ultrahighly titanium-doped silicon for the implementation of an intermediate band material," Applied Physics Letters, vol. 94, no. 4, Jan 26, 2009.

[13] D. Pastor, J. Olea, A. del Prado, E. Garcia-Hemme, R. Garcia-Hernansanz, and G. Gonzalez-Diaz, "Insulator to metallic transition due to intermediate band formation in Ti-implanted silicon," Solar Energy Materials and Solar Cells, vol. 104, pp. 159-164, Sep, 2012.

[14] E. Garcia-Hemme, R. Garcia-Hernansanz, J. Olea, D. Pastor, A. del Prado, I. Martil, and G. Gonzalez-Diaz, "Far infrared photoconductivity in a silicon based material: Vanadium supersaturated silicon," Applied Physics Letters, vol. 103, no. 3, Jul 15, 2013. 
[15] S. Hu, P. Han, S. Wang, X. Mao, X. Li, and L. Gao, "Improved photoresponse characteristics in Se-doped Si photodiodes fabricated using picosecond pulsed laser mixing," Semiconductor Science and Technology, vol. 27, no. 10, Oct, 2012.

[16] D. Pastor, J. Olea, A. del Prado, E. García-Hemme, I. Mártil, G. González-Díaz, J. Ibáñez, R. Cuscó and L. Artús, "UV and visible Raman scattering of ultraheavily $\mathrm{Ti}$ implanted $\mathrm{Si}$ layers for intermediate band formation", Semiconductors Science and Technology, vol. 26, 115003, Oct, 2011.

[17] M. A. Green, "Self-consistent optical parameters of intrinsic silicon at $300 \mathrm{~K}$ including temperature coefficients," Solar Energy Materials and Solar Cells, vol. 92, no. 11, pp. 1305-1310, Nov, 2008.

[18] S. Hocine, and D. Mathiot, "Titanium diffusion in silicon," Applied Physics Letters, vol. 53, no. 14, pp. 1269-1271, Oct 3, 1988.

[19] D. Mathiot, and S. Hocine, "Titanium-related deep levels in silicon - A reexamination," Journal of Applied Physics, vol. 66, no. 12, pp. 5862-5867, Dec $15,1989$.

[20] D. Pastor, J. Olea, A. Muñoz-Martín, A. Climent-Font, I. Mártil and G. González-Díaz, "Interstitial $\mathrm{Ti}$ for intermediate band formation in Tisupersaturated silicon", Journal of Applied Physics, vol. 112, 113514.1 - 5, (2012).

[21] J. Olea, G. Gonzalez-Diaz, D. Pastor, I. Martil, A. Marti, E. Antolin, and A. Luque, "Two-layer Hall effect model for intermediate band Ti-implanted silicon," Journal of Applied Physics, vol. 109, no. 6, 2011.

[22] J. Olea, D. Pastor, E. Garcia-Hemme, R. Garcia-Hernansanz, A. del Prado, I. Martil, and G. Gonzalez-Diaz, "Low temperature intermediate band metallic behavior in Ti implanted Si," Thin Solid Films, vol. 520, no. 21, pp. 6614-6618, Aug 31, 2012.

[23] A. Rohatgi, J. R. Davis, R. H. Hopkins, P. Raichoudhury, P. G. McMullin, and J. R. McCormick, "Effect of titanium, copper and iron on silicon solar-cells," Solid-State Electronics, vol. 23, no. 5, pp. 415-\&, 1980, 1980.

[24] See http://www.engineering.unsw.edu.au/energy-engineering/pc1d-software-formodelling-a-solar-cell.

[25] J. C. Fontaine, S. Barthe, J. P. Ponpon, J. P. Schunck, and P. Siffert, "A simple procedure based on the PCD method for determination of recombination lifetime and surface recombination velocity in silicon," Measurement Science \& Technology, vol. 5, no. 1, pp. 47-50, Jan, 1994.

[26] D. Baek, S. Rouvimov, B. Kim, T. C. Jo, and D. K. Schroder, "Surface recombination velocity of silicon wafers by photoluminescence," Applied Physics Letters, vol. 86, no. 11, Mar 14, 2005.

[27] A. W. Stephens, A. G. Aberle, and M. A. Green, "Surface recombination velocity measurements at the silicon-silicon dioxide interface by microwavedetected photoconductance decay," Journal of Applied Physics, vol. 76, no. 1, pp. 363-370, Jul 1, 1994.

[28] J. Olea, D. Pastor, A. del Prado, E. Garcia-Hemme, R. Garcia-Hernansanz, I. Martil, and G. Gonzalez-Diaz, "Ruling out the impact of defects on the below band gap photoconductivity of Ti supersaturated Si," Journal of Applied Physics, vol. 114, no. 5, Aug 7, 2013. 